$\stackrel{N}{N}$

Global Journals Inc.

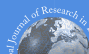

\title{
A Look at the Uncertainty of Measuring the Fundamental Constants and the Maxwell Demon from the Perspective of the Information Approach
}

By Boris Menin

Abstract- This paper proposes a new framework for calculating the discrepancy of a model and the observed technological process or physical phenomenon. It offers powerful tools for all measurement methods applied in technology, engineering and experimental physics. Since the studies that validate and verificate the models of the phenomenon are still complex, they need to be combined into one total measure. Existing methods used in almost all literature up to the present time implicitly suggest that the use of supercomputers and the latest mathematical statistical methods allows achieving high accuracy very close to the boundaries of Heisenberg principle. To compare methodologies for improving models, we propose a new metric called comparative uncertainty. This allows us to prove that there is a limit to the achievable discrepancy between the model and the object under study.

Keywords: bekenstein bound; fundamental physical constants; information theory; landauer limit; mathematical modeling; maxwell demon, similarity theory.

GJRE-A Classification: FOR Code: 091399

$\|||||||||$

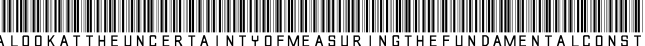

Strictly as per the compliance and regulations of:

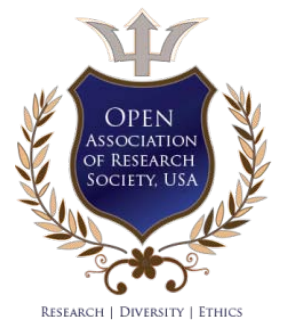

(C) 2019. Boris Menin. This is a research/review paper, distributed under the terms of the Creative Commons AttributionNoncommercial 3.0 Unported License http://creativecommons.org/licenses/by-nc/3.0/), permitting all non commercial use, distribution, and reproduction inany medium, provided the original work is properly cited. 


\title{
A Look at the Uncertainty of Measuring the Fundamental Constants and the Maxwell Demon from the Perspective of the Information Approach
}

\author{
Boris Menin
}

Abstract-This paper proposes a new framework for calculating the discrepancy of a model and the observed technological process or physical phenomenon. It offers powerful tools for all measurement methods applied in technology, engineering and experimental physics. Since the studies that validate and verificate the models of the phenomenon are still complex, they need to be combined into one total measure. Existing methods used in almost all literature up to the present time implicitly suggest that the use of supercomputers and the latest mathematical statistical methods allows achieving high accuracy very close to the boundaries of Heisenberg principle. To compare methodologies for improving models, we propose a new metric called comparative uncertainty. This allows us to prove that there is a limit to the achievable discrepancy between the model and the object under study. Our results have wide implications for known climate forecasts, spacecraft missions, measurement of fundamental constants, and other measurements during any technological processes. In this paper, the use of the information approach is illustrated by several examples: measurement of fundamental constants and calculation of the amount of information received by the Maxwell demon during modelling. In addition, we apply the Landauer limit to calculate the amount of information corresponding to the energy contained in the universe, with a known radius.

Keywords: bekenstein bound; fundamental physical constants; information theory; landauer limit; mathematical modeling; maxwell demon, similarity theory.

\section{INTRODUCTION}

J hysical laws express in mathematical form a quantitative relationship between different physical quantities. They are based on the generalization of the experimental data obtained and reflect the objective laws that exist in nature. It is so fundamentally important that all physical laws are an approximation to reality, since the construction of theories is formulated by certain models of phenomena and processes. Beyond these models, laws do not work or work poorly. Therefore, laws have certain limits of applicability. In other words, physical laws give good predictions in a specific area of experimental conditions, and the

Author: Mechanical \& Refrigeration Consultation Expert, Beer-Sheba, 8464209, Israel. e-mail: meninbm@gmail.com corresponding theory explains them. A more accurate or more correct theory has a wider range of applications. Scientists believe that physical laws, at least, allow us to predict the results with arbitrary accuracy. For example, classical mechanics, based on the three laws of Newton and the law of universal gravitation, is valid only for the motion of bodies with velocities much less than the speed of light. If the velocities of bodies are comparable with the speed of light, the predictions of classical mechanics are erroneous. The special theory of relativity has successfully coped with these problems. In fact, all physical theories are limited. The principle of correspondence requires that the new theory with a wider scope of applicability be limited to the old theory within its applicability. An appeal to the theory of new concepts creates important prerequisites for further development.

Among the various explanations for the admissibility of possible limits of applicability of physical laws, the following are most often used. The first is the assumption that there is a limited destabilization of phenomena for which the Heisenberg inequality gives a quantitative expression. Secondly, the limitations are determined by the real nature of the macroscopic instrument or measuring system. Most of the devices are finally presented as a solid one. In principle, it can be argued that any device has an educational effect only in the realm of reality, what it is. Thus, the results of research should be expressed in terms of macroscopic. In other words, concepts and images can be identified and associated only with ordinary macroscopic representations. The last argument is the point of view of the principle of the electromagnetic nature of all modern means of measurement and their role in determining the limits of experimental and measurement capabilities and harmonizing data with theoretical postulates. Thus, explanations are possible, but any quantitative approaches to quantifying the difference between the model (formulated by the physical law) and the existing reality have not been proposed to date.

Concerning the fundamental physical constants, it should be noted that their values are the accuracy of our knowledge of the fundamental 
properties of matter. On the one hand, very often the verification of physical theories is determined by the accuracy of the measured physical constant. On the other hand, firmly established experimental data form the basis of new physical theories.

When studying physical constants, it should be noted that they are measured with very high accuracy, which is steadily increasing. In itself it is evidence of the development and improvement of methods of physical experiment. Nowadays, exact research is carried out to measure and refine the values of physical constants and to work diligently to harmonize data obtained by different methods and different groups of researchers. However, there is an urgent need to further improve the accuracy of measuring fundamental physical constants. This is explained by the desire to improve the axiomatic basis of the International System of Units (SI).

To estimate the accuracy achieved in various nature and technological processes, including measurements of fundamental physical constants, the concept of relative uncertainty is used. It should be noted that this method of determining the measurement accuracy does not indicate the direction in which the true value of the fundamental physical constant can be found. In addition, it includes an element of subjective judgment [1].

In [2], the authors proposed to simplify the method for estimating the uncertainties of the measurement results: provided that the indirectly measured physical quantity depends only on the directly measured physical quantities directly or indirectly, the evaluation of the measurement accuracy using the maximum uncertainty can be interpreted as having a much a higher informative value than the value provided by a simple orientation estimate.

On the contrary, we propose a new method for estimating the reliability of the obtained measurement results by achieving the least relative uncertainty.

Taking into account the aforesaid, the use of information theory for the modelling of physical processes takes on a special place. Informationoriented theoretical calculations of models of physical phenomena are based on the analogy between measurement systems and communication systems. In a simple communication system, the message (input) is encoded into a signal at the end of the transmitter, sent to the end of the receiver, and then decoded back (output). The accuracy of the transmission depends on the characteristics of the communication system, as well as on the characteristics of the medium, that is, on the background noise level. Similarly, measuring instruments can be considered as "information machines" [3], which interact with the object in this state (input), code this state into an internal signal and convert this signal into readout (output). The accuracy of the measurement is similarly dependent on the instrument, as well as on the noise level in its environment. Conceived as a special type of information transfer, the dimension is analysed from the point of view of the conceptual apparatus of information theory [4].

Bas van Fraassen [5] recently proposed a measurement concept in which information plays a key role. He considers measurements as consisting of two levels: at the physical level, the measuring device interacts with the object and reads, for example, the position of the pointer. At the abstract level, this theory represents the possible states of the object in the parameter space. Measurement finds an object in the sub-region of this space of abstract parameters, thereby reducing the range of possible states. This reduction in capabilities is reduced to collecting information about the measured object.

Since the studies that confirm and test the phenomenon models are still complex, they need to be combined into one general measure. The methods used in almost all literature up to the present time implicitly suggest that the use of supercomputers and the latest mathematical statistical methods makes it possible to achieve a high arbitrary accuracy very close to the boundaries of the Heisenberg principle. To compare the methodologies for improving models, we propose a new metric called comparative uncertainty. This metric is a novel one. This allows us to prove that there is a limit to the achievable discrepancy between the model and the object under study. In another words, the state of an object cannot be known with arbitrary precision independently of its measurement [6].

Here we investigate the information cost of measurements in the modelling. Starting with the framework set in ref. [7], we introduce the metric of dignity, the comparative uncertainty of the measurement, which is realized in a real experiment. We believe in the importance of this work because of the following points. Thanks to the introduction of this quantitative tool, we obtain the lower limit of the achieved absolute and relative uncertainties associated with the act of observation, which is characteristic and inherent in measurement. The flexibility of our experimental setup allows us to calculate the amount of information retrieved from the system. Our method allows us to determine how much the developed model (before carrying out the experiment or computer calculations) can extract information in order to achieve the lowest threshold inconsistency in comparison with the object under study. Moreover, there was showed that the information amount inherent in the model can be calculated and how it proscribes the required number of quantities which should be taken into account. It was thus concluded that in most physically relevant cases (micro- and macro-physics), the comparative uncertainty can be realized by field tests or computer simulations within the prearranged variation of the main recorded quantity. 
The information-oriented approach was applied for design of thermal energy storage systems [8], technological processes of slurry ice production [9], climate models and spacecraft heating [10]. Since the information approach provides a theoretically grounded value of relative uncertainty, with great certainty, one can estimate the admissibility of a particular measurement result. It can also be easily updated when new measurements come out. The approach can also be used for the measurements of the fundamental physical constants and will greatly shorten the duration of the studies and the design stage, thereby reducing the cost of the projects. This moment, it will be revised for a very controversial, at the current level of understanding nature by scientists, measurements of Hubble constant and concept of the Maxwell's demon.

Our work is organized as follows. In the next section, we introduce an information-oriented approach and a methodology for calculating relative and comparative uncertainties. In Section III, we use the information method to analyze the relative uncertainties of the Hubble, Boltzmann, Planck, fine structure, and gravitational constants. In addition, we analyze the paradox of Maxwell's demon and we apply the Landauer limit to calculate the amount of information corresponding to energy contained in the universe, with a known radius. We discuss in Section 4 and conclude in Section 5.

\section{il. Key Concepts from Information- Oriented Approach}

In seminal paper [7] the approach, called $\mu_{\mathrm{SI}}$ - hypothesis, for calculating the lowest comparative uncertainty of the researched quantity based on principles of information and similarity theories with the usage of the International system of units (SI), is formulated. Following it, the certain uncertainty exists before starting experiment due only the known recorded number of quantities. In turn, the dimensionless comparative uncertainty $\varepsilon$ of the dimensionless quantity $u$, which varies in a predetermined dimensionless interval $S$, for a given number of selected physical dimensional quantities $z^{\prime \prime}$, and $\beta^{\prime \prime}$ (the number of the recorded base quantities) can be determined from the relation:

$$
\varepsilon=\Delta u / S \leq\left[\left(z^{\prime}-\beta^{\prime}\right) /(\Psi-\xi)+\left(z^{\prime \prime}-\beta^{\prime \prime}\right) /\left(z^{\prime}-\beta^{\prime}\right)\right]
$$

where $\Delta u$ is the dimensionless uncertainty of physicalmathematical model describing the experiment of measurement of the dimensionless quantity u;

$\xi$ is a number of the base quantities with independent dimension; SI includes the following seven $(\xi=7)$ base primary quantities: $L$ is the length, $M$ is weight, $T$ is time, $I$ is electric current, $\Theta$ is thermodynamic temperature, $J$ is force of light, $F$ is a number of substances. The dimension of any derived quantity $q$ can only express a unique combination of dimensions of base quantities in different degrees [11]:

$$
\boldsymbol{q} \supset \boldsymbol{L}^{\prime} \boldsymbol{M}^{m} \boldsymbol{T}^{t} \boldsymbol{I}^{i} \Theta^{\Theta} \boldsymbol{J}^{j} \boldsymbol{F}^{f} .
$$

$I, m \ldots f$ are exponents of quantities, the range of each has maximum and minimum value; according to [12], integers are the following:

$$
\begin{gathered}
-3 \leq I \leq+3,-1 \leq m \leq+1,-4 \leq t \leq+4,-2 \leq i \leq+2, \\
-4 \leq \Theta \leq+4,-1 \leq j \leq+1,-1 \leq f \leq+1
\end{gathered}
$$

The exponents of quantities take only integer values [12], so the number of choices of dimensions for each quantity $e_{k}, k=\{l, m \ldots f\}$ according to (3) is the following:

$$
e_{l}=7 ; e_{m}=3 ; e_{t}=9 ; e_{i}=5 ; e_{\theta}=9 ; e_{j}=3 ; e_{f}=3 ;
$$

where, for example, $L^{-3}$ is used in a formula of density, and $\Theta^{4}$ in the Stefan-Boltzmann law.

The total number of dimension options of physical quantities equals $\boldsymbol{\Psi}^{*}=\prod_{l}^{f} \boldsymbol{e}_{\boldsymbol{k}}-1$

$$
\psi^{\star}=e_{l} e_{m} e_{t} e_{i} e_{\theta} e_{j} e_{f}-1=7 \cdot 3 \cdot 9 \cdot 5 \cdot 9 \cdot 3 \cdot 3-1=76,544,
$$

where "-1" corresponds to the occasion when all exponents of base quantities in the formula (2) are treated to zero dimension; $\Pi$ is a product of $e_{k}$;

The value $\psi^{\star}$ includes both required, and inverse quantities (for example, $L^{1}$ - length, $L^{-1}$ - running length). The object can be judged knowing only one of its symmetrical parts, while others structurally duplicating this part may be regarded as information empty [13]. Therefore, the number of options of dimensions may be reduced in 2 times. It means that the total number of dimensional physical quantities without inverse quantities for SI equals.

$$
\psi=\psi^{\star} / 2=38,272
$$

$z^{\prime}$ is a total number of dimensional physical quantities in the chosen class of phenomena (COP); in SI frames, every researcher selects a particular COP to study material object. COP is a set of physical phenomena and processes described by a finite number of base and derived quantities that characterize certain features of material object from the position with qualitative and quantitative aspects [14]. In studying mechanics, for example, which widely applied for the Newtonian gravitational constant measurements with a torsion balance, the base units of SI are typically used: $L, \quad M, T(L M T)$. In publications relating to the measurement, for example, of the Boltzmann constant, the model corresponds to $\mathrm{COP}_{\mathrm{SI}} \equiv \angle M T \theta$;

$\beta^{\prime}$ is the number of base physical quantities in the chosen COP; taking into accountr -theorem [11], a total number of dimensionless criteria $\mu_{\mathrm{SI}}=\psi-\xi$ inherent in SI equals.

$$
\mu_{\mathrm{SI}}=\psi-\xi=38,265 .
$$


Equation (1) quantifies $\Delta u / S$ caused by the limited number of quantities taken into account in the theoretical or experimental analysis of researched quantity. On the other hand, it also sets a limit on the expedient increasing of the measurement accuracy in conducting experimental studies. In turn, $\Delta u / S$ is not a purely mathematical abstraction. It has a physical meaning, consisting in the witness that in nature there is a fundamental limit to the accuracy of displaying any observed material object, which cannot be surpassed by any improvement of instruments and methods of measurement. The reality of the environment is the obvious a priori condition for the modeling of the investigated material object. By allocating the interested process or phenomenon, the unknown relationships between the content of object and the environment are "broken". In this context it is obvious that an overall uncertainty of the model including inaccurate input data, physical assumptions, the approximate solution of the integral-differential equations, etc., will be larger than $\Delta u$. Thus, $\Delta u$ is only one lowest component of a possible mismatch of real object and its modeling results.

In fact, equation (1) can be regarded as the conformity principle (uncertainty relation) for the process of model development. No model can produce results that contradict the relation (1). That is, any change in the level of the detailed description of the observed object $\left(z^{\prime \prime}-\beta^{\prime \prime} ; \quad z^{\prime}-\beta^{\prime}\right)$ causes a change in the minimum comparative uncertainty value $\Delta_{\text {pmm }} / S$ of the model of a specific COP and in the achieved accuracy of each main quantity, characterizing the internal structure of the object. In other words, the conformity principle fundamentally establishes the accuracy limit (for a given class of phenomena) of simultaneously defining a pair of quantities, observed by a conscious researcher, particularly, the absolute uncertainty in the measurement of the investigated quantity and the interval of its changes.

Equating the derivative of $\Delta u / S$ (1) with respect to $z^{\prime}-\beta^{\prime}$, to zero, we obtain the condition for achieving the minimum comparative uncertainty for a particular COP:

$$
\left(z^{\prime}-\beta^{\prime}\right)^{2} /(\Psi-\xi)=\left(z^{\prime \prime}-\beta^{\prime \prime}\right)
$$

It should be noted that, for example, for the electromagnetism processes $\left(\mathrm{COP}_{\mathrm{SI}} \equiv L M T\right)$, which are used usually for the Rydberg constant measurements, the lowest comparative uncertainty $\varepsilon_{\mathrm{LMTl}}$ can be reached at the following conditions:

$$
\begin{gathered}
\left(z^{\prime}-\beta^{\prime}\right)=\left(e_{l} e_{m} e_{t} e_{i}-1\right) / 2-4=(7 \cdot 3 \cdot 9 \cdot 5-1) / 2-4=468 \\
\left(z^{\prime \prime}-\beta^{\prime \prime}\right)=\left(z^{\prime}-\beta^{\prime}\right)^{2} /(\Psi-\xi)=468^{2} / 38,265 \approx 6
\end{gathered}
$$

Then one can calculate an achievable comparative uncertainty $\varepsilon_{\mathrm{LMTI}}$

$$
\varepsilon_{\text {LMTI }}=(\Delta U / S)_{L M T I}=468 / 38,265+6 / 468 \approx 0.0244
$$

Below is Table 1 introducing different class of phenomena and the corresponding achievable comparative uncertainties and recommended number of quantities.

Let to apply the above-mentioned method to determine the minimum possible measurement uncertainty of several fundamental physical constants.

Table 1: Comparative uncertainties and recommended number of dimensionless criteria

\begin{tabular}{|c|c|c|}
\hline COP $_{\mathrm{SI}}$ & Comparative uncertainty & Number of criteria \\
\hline$L M T$ & 0.0048 & $0.2<1$ \\
\hline$L M T F$ & 0.0146 & $\cong 2$ \\
\hline$L M T I$ & 0.0244 & $\cong 6$ \\
\hline$L M T \theta$ & 0.0442 & $\cong 19$ \\
\hline$L M T I F$ & 0.0738 & $\cong 52$ \\
\hline$L M T \theta F$ & 0.1331 & $\cong 169$ \\
\hline$L M T \theta I$ & 0.2220 & $\cong 471$ \\
\hline LMTOFI & 0.6665 & $\cong 4,249$ \\
\hline
\end{tabular}

For these purposes the reader needs to remember that if the range of observation $S$ is not defined, the information obtained during the observation or measurement cannot be determined, and the entropic price becomes infinitely large [15]. In the framework of the information-oriented approach, it seems that the theoretical limit of the absolute and relative uncertainties depends on the empirical value, that is, possible interval of placing (the observed range of variations) $S$ of the measured physical constant. In other words, the results will be completely different if a larger interval of changes is considered in the measured fundamental physical constant. It is right, however, if $S$ is not declared, the information obtained in the measurement cannot be determined. Any specific measurement requires certain (finite) a priori information about the components of the measurement and interval of observation of the measured quantity. These requirements are so universal that it acts as a postulate of metrology [16]. This, the observed range of variations, depends on the knowledge of the developer before undertaking the study. "If nothing is known about the system studied, then $S$ is determined by the limits of the measuring devices used" [15].

That is why, taking into account Brillouin's suggestions, there are two options of applying the conformity principle to analyze the measurement data of the fundamental physical constants.

First, this principle dictates, factually, analyzing the data of the magnitude of the achievable relative uncertainty at the moment taking into account the latest results of measurements. The extended range of changes in the quantity under study $S$ indicates an imperfection of the measuring devices, which leads to a large value of the relative uncertainty. The development of measuring technology, the increase in the accuracy of measuring instruments, and the improvement in the existing and newly created measurement methods 
together lead to an increase in the knowledge of the object under study and, consequently, the magnitude of the achievable relative uncertainty decreases. However, this process is not infinite and is limited by the conformity principle. The reader should bear in mind that this conformity principle is not a shortcoming of the measurement equipment or engineering device, but of the way the human brains work. When predicting behavior of any physical process, physicists are, in fact, predicting the perceivable output of instrumentation. It is true that, according to the $\mu$-hypothesis, observation is not a measurement, but a process that creates a unique physical world with respect to each particular observer. Thus, in this case, the range of observation (possible interval of placing) of the fundamental physical constant $S$ is chosen as the difference between the maximum and minimum values of the physical constant measured by different scientific groups during a certain period of recent years. Only in the presence of the results of various experiments one can speak about the possible appearance of a measured value in a certain range. Thus, using the smallest attainable comparative uncertainty inherent in the selected class of phenomena during measuring the fundamental constant, it is possible to calculate the recommended minimum relative uncertainty that is compared with the relative uncertainty of each published study. In what follows, this method is denoted as IARU and includes the following steps:

1. From the published data of each experiment, the value $z$, relative uncertainty $r_{z}$ and standard uncertainty $u_{z}$ (possible interval of $u$ placing) of the fundamental physical constant are chosen.

2. The experimental absolute uncertainty $\Delta_{z}$ is calculated by multiplying the fundamental physical constant value $z$ and its relative uncertainty $r_{z}$ attained during the experiment, $\Delta_{z}=z \cdot r_{z}$.

3. The maximum $z_{\max }$ and minimum $z_{\min }$ values of the measured physical constant are selected from the list of measured values $z_{i}$ of the fundamental physical constant mentioned in different studies.

4. As a possible interval for placing the observed fundamental constant $S_{z}$, the difference between the maximum and minimum values is calculated, $S_{z}=z_{\max }-z_{\min }$.

5. The selected comparative uncertainty $\varepsilon_{T}$ (Table 1) inherent in the model describing the measurement of the fundamental constant is multiplied by the possible interval of placement of the observed fundamental constant $S_{z}$ to obtain the absolute experimental uncertainty value $\Delta$ IARU in accordance with the IARU, $\Delta_{\text {IARU }}=\varepsilon_{T} \cdot S_{z}$.

6. To calculate the relative uncertainty $r_{\text {IARU }}$ in accordance with the IARU, this absolute uncertainty $\Delta_{\text {IARU }}$ is divided by the arithmetic mean of the selected maximum and minimum values, $r_{\text {IARU }}=\Delta_{\text {IARU }} /\left(\left(z_{\max }+z_{\text {min }}\right) / 2\right)$.

7. The relative uncertainty obtained $r_{\text {IARU }}$ is compared with the experimental relative uncertainties $r_{i}$ achieved in various studies.

8. According to $I A R U$, a comparative experimental uncertainty of each study, $\varepsilon_{\text {IARUi }}$ is calculated by dividing the experimental absolute uncertainty of each study $\Delta_{z}$ on the difference between the maximum and minimum values of the measured fundamental constant $S_{z}, \varepsilon_{\text {IARUi }}=\Delta_{z} / S_{z}$. These calculated comparative uncertainties are also compared with the selected comparative uncertainty $\varepsilon_{\mathrm{T}}$ (Table 1).

Second, $S$ is determined by the limits of the measuring devices used [15]. This means that as the observation interval in which the expected true value of the measured fundamental physical constant is located, a standard uncertainty is selected when measuring the physical constant in each particular experiment. Compared with various fields of technology, experimental physics is better for the fact that in all the researches, the experimenters introduce the output data of the measurement with uncertainty bars. At the same time, it should be remembered that the standard uncertainty of a particular measurement is subjective, because the conscious observer probably did not take into account this or that uncertainty. The experimenters calculate the standard uncertainty, taking into account all possibilities, they noticed the measured uncertainties. Then, one calculates ratio between the absolute uncertainty reached in an experiment and standard uncertainty, acting as a possible interval for allocating a fundamental physical constant. So, in the framework of the information approach, the comparative uncertainties achieved in the studies are calculated, which in turn are compared with the theoretically achievable comparative uncertainty inherent in the chosen class of phenomena. Standard uncertainty can be calculated also for quantities that are not normally distributed. Transformation of different types of uncertainty sources into standard uncertainty is very important. In what follows, this method is denoted as IACU and includes the following steps:

1. From the published data of each experiment, the value $z$, relative uncertainty $r_{z}$ and standard uncertainty $u_{z}$ (possible interval of placing) of the fundamental physical constant are chosen.

2. The experimental absolute uncertainty $\Delta_{z}$ is calculated by multiplying the fundamental physical constant value $z$ and its relative uncertainty $r_{z}$ attained during the experiment, $\Delta_{z}=z \cdot r_{z}$.

3. The achieved experimental comparative uncertainty of each published research $\varepsilon_{\text {IACUi }}$ is calculated by dividing the experimental absolute uncertainty $\Delta_{z}$ on the standard uncertainty $u_{z}, \varepsilon_{I A C U i}=\Delta_{z} / u_{z}$.

\section{}


4. The experimental calculated comparative uncertainty $\varepsilon_{\text {IACUi }}$ is compared with the selected comparative uncertainty $\varepsilon_{\mathrm{T}}$ (Table 1 ) inherent in the model, which describes the measurement of the fundamental constant.

\section{ili. Applications}

On the one hand, equation (1) requires the use of an experimental stand with the number of variables corresponding to the chosen class of phenomena. On the other hand, equation (1) clearly indicates the impossibility to develop an experimental device that allows to achieve the exact value of the selected comparative uncertainty for a given measurement result. Its introduction emphasizes the need for the development of new experimental stands suitable for quantifying the quantity under study. The $\mu_{\mathrm{SI}}$-hypothesis given in equation (1) makes the lower bound of the change in the entropy of the chosen model inaccessible from theoretical considerations. Our experiment allows us to estimate the comparative uncertainty $\Delta u$ / $S$ from the published results, although this is not equivalent to measuring the actual changes in the fundamental constant. This trend is reflected in the spread of the value of comparative uncertainty in comparison with its theoretical-informational lower limit, depending on the chosen class of phenomena.

a) Hubble constant $H_{0}$

The current state of Hubble's constant $\mathrm{H}_{0}$ definitions gives the scale of the length of the universe, connecting the speed of expansion of objects with their distance. There are two broad categories of measurements. In the first case $(\Lambda$ Cold Dark Matter model - $\Lambda$ CDM), individual astrophysical objects are used that have some property that allows them to determine their internal brightness or size or allows them to determine their distance geometrically. The second category includes the use of a cosmic microwave background $(\mathrm{CMB})$ in the sky, or the correlation between large samples of galaxies, to determine information about the geometry of the universe and, consequently, the Hubble constant, usually in combination with other cosmological parameters. The current results give a range of the Hubble constant changes from $67 \mathrm{~km} \cdot \mathrm{s}^{-1} \cdot \mathrm{Mpc}^{-1}$ to $74.3 \mathrm{~km} \cdot \mathrm{s}^{-1} \cdot \mathrm{Mpc}^{-1}$. Whether there is a discrepancy and whether a new physics is needed to solve it depends on details of the systematic of objectoriented methods, as well as on assumptions about other cosmological parameters and on what data sets are combined in the case of sky methods. Maybe the Hubble constant discrepancy is just due unaccounted uncertainties.

Of course, all these measurements have uncertainty. Each research team first produces unprocessed measurements, then attempts to explain the vagaries of individual telescopes, astrophysical unknowns and countless other sources of uncertainty that can hold astronomers all day. Then, all the individual published studies are combined into a single number for the rate of expansion, as well as a measure of how vague this number is.

Several research publications made during 2009-2018 [17-25] were analysed from the position of the introduction of clear achieved values of the relative uncertainty with a published possible interval of $\mathrm{HO}$ placing. Comparative uncertainties in the measurement of the Hubble constant are calculated. The reader must bear in mind that measurements closed to $\Lambda \mathrm{CDM}$ belong $\mathrm{COP}_{\mathrm{SI}} \equiv L M T \theta$ and the Hubble constant measurements closed to $\mathrm{CMB}$ belong to $\mathrm{COP}_{\mathrm{SI}} \equiv L M T$. In the framework of the information-oriented approach it is impossible not only to achieve, but also to approach the smallest relative uncertainty when using $\mathrm{COP}_{\mathrm{SI}} \equiv L M T$ (see Table 1): the optimal number of the dimensionless criteria is less 1 . That is why, further calculations will be carried out only for $\mathrm{COP}_{\mathrm{SI}} \equiv L M T \theta$.

In order to apply the stated approach (IARU), as the possible measurement interval $\mathrm{H}_{0}$, there was chosen the difference in its values obtained in two projects: $\mathrm{H}_{0 \min }=67.0 \mathrm{~km} \cdot \mathrm{C}-1 \cdot \mathrm{Mpc}^{-1}$ [25] and $\mathrm{H}_{0 \max }=72.5 \mathrm{~km}$. $\mathrm{C}^{-1} \cdot \mathrm{Mpc}^{-1}[21]$.

Then the possible observable range $S_{H}$ of changes $\mathrm{H}_{0}$ and the average value $\mathrm{H}_{\text {oaver }}$ are equal:

$$
\begin{gathered}
\boldsymbol{S}_{\mathrm{H}}=\mathrm{H}_{0 \max }-\mathrm{H}_{0 \min }=5.5\left(\mathrm{~km} \cdot \mathrm{c}^{-1} \cdot \mathrm{Mpc}^{-1}\right) \\
\left(\mathrm{H}_{0}\right)_{\mathrm{aver}}=\left(\mathrm{H}_{0 \max }+\mathrm{H}_{0 \min }\right) / 2=69.75\left(\mathrm{~km} \cdot \mathrm{c}^{-1} \cdot \mathrm{Mpc}^{-1}\right) .
\end{gathered}
$$

Applying the IARU approach, one can calculate the desired value of the relative uncertainty $\left(r_{H}\right)_{L M T \theta}$, by which the researchers in the future are going to

$$
\left(\varepsilon_{\mathrm{H}}\right)_{\text {LMT } \theta}=0.0442 \text {. }
$$

Then an absolute uncertainty $\left(\Delta_{H}\right)_{\llcorner M T \theta}$ for $\mathrm{COP}_{\mathrm{SI}} \equiv L M T \theta$.

$$
\left(\Delta_{\mathrm{H}}\right)_{L M T \theta}=\left(\varepsilon_{\mathrm{H}}\right)_{L M T \theta} \cdot S_{\mathrm{H}}=0.0442 \cdot 5.5=0.243199\left(\mathrm{~m}^{-1}\right) .
$$


Table 2: Hubble constant determinations and relative and comparative uncertainties achieved

\begin{tabular}{|c|c|c|c|c|c|c|c|}
\hline \multirow[t]{2}{*}{ Year } & $\begin{array}{c}\text { Hubble } \\
\text { constant }\end{array}$ & $\begin{array}{l}\text { Achieved } \\
\text { relative } \\
\text { uncertainty }\end{array}$ & $\begin{array}{l}\text { Absolute } \\
\text { uncertainty }\end{array}$ & $\begin{array}{c}\mathrm{H}_{0} \text { possible } \\
\text { interval of } \\
\text { placing }\end{array}$ & $\begin{array}{l}\text { Calculated } \\
\text { comparative } \\
\text { uncertainty }\end{array}$ & $\begin{array}{l}\text { Calculated } \\
\text { comparative } \\
\text { uncertainty }\end{array}$ & \multirow{2}{*}{ Ref } \\
\hline & $\frac{\mathrm{H}_{0}}{\mathrm{~km} \cdot \mathrm{c}^{-1} \cdot \mathrm{Mpc}^{-1}}$ & $r_{H}$ & $\frac{\Delta_{H}}{\mathrm{~km} \cdot \mathrm{c}^{-1} \cdot \mathrm{Mpc}^{-1}}$ & $\frac{\mathrm{u}_{\mathrm{H}}}{\mathrm{km} \cdot \mathrm{c}^{-1} \cdot \mathrm{Mpc}^{-1}}$ & $\begin{array}{c}\varepsilon_{\mathrm{H}^{\prime}}=\Delta_{\mathrm{H}} / \mathrm{u}_{\mathrm{H}} \\
I A C U\end{array}$ & $\begin{array}{c}\varepsilon_{\mathrm{H}^{\prime}}=\Delta_{H} / \mathrm{S}_{\mathrm{H}} \\
\text { IARU }\end{array}$ & \\
\hline 2009 & 71.9 & 0.038 & 2.7 & $5.2^{\star \star}$ & 0.5192 & 0.49 & [17] \\
\hline 2011 & 72.0 & 0.042 & 3.0 & $4.7^{\star}$ & 0.6383 & 0.55 & [18] \\
\hline 2011 & 71.0 & 0.035 & 2.5 & $6.0^{\star \star \star}$ & 0.4167 & 0.45 & [19] \\
\hline 2013 & 69.32 & 0.012 & 0.8 & $4.3^{\star \star}$ & 0.1860 & 0.15 & [20] \\
\hline 2014 & 72.5 & 0.034 & 2.5 & $6.5^{\star}$ & 0.3846 & 0.45 & [21] \\
\hline 2014 & 69.6 & 0.010 & 0.7 & $1.4^{*}$ & 0.5000 & 0.13 & [22] \\
\hline 2015 & 68.11 & 0.013 & 0.86 & $1.4^{\star}$ & 0.6143 & 0.16 & [23] \\
\hline 2016 & 67.8 & 0.013 & 0.9 & $2.2^{\star \star}$ & 0.4091 & 0.16 & [24] \\
\hline 2018 & 67.0 & 0.013 & 0.9 & $1.0^{\star \star}$ & 0.9000 & 0.16 & [25] \\
\hline
\end{tabular}

* Data are introduced in [23];

** Data are introduced in [26]

In this case, the lowest relative uncertainty $r_{\text {LMTO }}$ for $\mathrm{COP}_{\mathrm{SI}} \equiv L M T \theta$ is as follows:

$$
\boldsymbol{r}_{\text {LMT } \theta}=\boldsymbol{\Delta}_{\text {LMT } \theta} /\left(\left(\mathrm{H}_{\max }+\mathrm{H}_{\min }\right) / 2\right)=0.0035 \text {. }
$$

An analysis of the data given in Table 2 [17-25], shows that during the last decade there has been sharp increase in the accuracy of measuring $\mathrm{H}_{0}$ from the point of view of reducing the relative uncertainty.

Following the method IARU, it is seen from the data given in Table 2 that there was too a significant improving the accuracy of the measurement of the Hubble constant during last decade. It is authorized true when based on calculation of the relative and comparative uncertainties. Judging the data by the comparative uncertainty following to IACU, one can see that the measurement accuracy was not improved. At the same time, there is yet a large gap between the comparative uncertainty calculated according to the information-oriented approach $\varepsilon_{\text {LMTO }}=0.0442$ and the experimental magnitudes achieved during measuring $\mathrm{H}_{0}$. It must be mentioned that, most likely, the exactness of Hubble constant as other fundamental physical constants, cannot be infinite. Therefore, the development of a larger number of designs and improvement of the various experimental facilities for the measurement of Hubble constant is an absolute must. In addition, the difference may be explained by the fact that experimenters take into account a very contrast number of quantities in comparison with the formulated number according to the information-oriented approach (Table 1).

Despite the very depressing situation revealed by using the information-oriented method to estimate the accuracy of the Hubble constant measurement, being optimistic, we can hope that in the near future scientists will be able to improve the measurement technique. On the other hand, as the analysis presented in the next chapter will show, measurements of other fundamental constants are performed with much higher accuracy, and the results of experimental studies coincide with the values recommended in the framework of the information approach.

b) Analysing publications of measurements of $k_{b}, h, N_{A}$, $\alpha^{-1}$ and $G$

Taking into account the methodology mentioned in Key concepts from information-oriented approach, there were analysed the measurement results of Boltzmann constant, Planck constant, Avogadro constant, inverse fine structure constant and gravitational constant published in different journals and CODATA recommendations. Data inherent in only relative uncertainties calculated according to IARU are introduced in Table 3[27].

Table 3: Fundamental physical constants: recommended and calculated relative uncertainties

\begin{tabular}{|c|c|c|c|c|c|c|}
\hline $\begin{array}{c}\text { Fundamental } \\
\text { constant }\end{array}$ & Designation & Dimension & $\begin{array}{c}\text { Class of } \\
\text { phenomena }\end{array}$ & $\begin{array}{c}\text { The analyzed } \\
\text { interval of } \\
\text { publications }\end{array}$ & $\begin{array}{c}\text { Published, } \\
\text { recommended relative } \\
\text { uncertainty }\end{array}$ & $\begin{array}{c}\text { Calculated relative } \\
\text { uncertainty } \\
(I A R U)\end{array}$ \\
\hline Boltzmann constant & $k_{\mathrm{b}}$ & $\mathrm{m}^{2} \cdot \mathrm{kg} \cdot \mathrm{c}^{-2} \cdot \mathrm{K}^{-1}$ & $L M T \theta F$ & $2015-2018$ & $3.7 \cdot 10^{-7}[28]$ & $2.8 \cdot 10^{-7}$ \\
\hline Plank constant & $\mathrm{h}$ & $\mathrm{m}^{2} \cdot \mathrm{kg}^{-2}$ & $L M T I$ & $2007-2014$ & $9.1 \cdot 10^{-9}[29]$ & $8.7 \cdot 10^{-9}$ \\
\hline Avogadro constant & $\mathrm{N}_{\mathrm{A}}$ & $\mathrm{mol}^{-1}$ & $L M T F$ & $2001-2015$ & $2 \cdot 10^{-8}[30]$ & $1.7 \cdot 10^{-8}$ \\
\hline $\begin{array}{c}\text { Inverse fine structure } \\
\text { constant }\end{array}$ & $\alpha^{-1}$ & & $L M T$ & $2006-2014$ & $2.9 \cdot 10^{-11}[31]$ & $2.9 \cdot 10^{-11}$ \\
\hline Gravitational constant & $\mathrm{G}$ & $\mathrm{m}^{3} \cdot \mathrm{kg}^{-1} \cdot \mathrm{c}^{-2}$ & $L M T I$ & $2000-2016$ & $4.7 \cdot 10^{-5}[32]$ & $1.35 \cdot 10^{-5}$ \\
\hline
\end{tabular}


It is seen from Table 3 that published recommended values of relative uncertainties are consistent with the same calculated according to IARU. This fact confirms that the theoretically grounded information approach allows for crafting of a meaningful picture of future results. However, the quantity that need to be predicted is generally not experimentally observable before the prediction, since otherwise no prediction would be needed. Assessing the credibility of such extrapolative predictions is challenging. In validation CODATA's approach, the model outputs for observed quantities are constructed, using modern advanced Bayesian statistical methods and powerful computers to determine if they are consistent. By itself, this consistency only ensures that the model can predict the measured physical constants under the conditions of the observations [33]. This limitation dramatically reduces the utility of the CODATA's effort for decision making because it implies nothing about predictions for scenarios outside of the range of observations. So, these remarks define the main principal difference between CODATA's approach and the informationbased and theoretically grounded method.

It should be noted that the proposed method is not considered universal, since it does not answer the question of choosing specific physical quantities for better representation of the surrounding world. The information-oriented approach for estimating the model's uncertainty does not involve a spatio-temporal or causal relationship between the quantities involved. Instead, it takes into account only the differences between their numbers. However, this method can be firmly asserted that this method reveals an inconsistency between the various methods of measuring a particular fundamental constant $\mathrm{H}_{0}$.

\section{c) Maxwell demon}

Over the past twenty years, both information in the form of a certain substance, and methods of information theory are the subject of special attention of scientists, engineers and philosophers. A great number of studies are devoted not only to clarifying the internal content of the concept of INFORMATION, but also to the application of this unique substance in all fields of human activity: physics, chemistry, biology, psychology, business, etc. The number of theories offered is uncountable. Impressive practical results were obtained using information theory in the field of quantum mechanics, telecommunications, medicine, marketing and the development of non-lethal weapons. At the same time, in theoretical and experimental physics, the number of research papers (with a specific quantitative result) using information theory is catastrophically small; they can be counted on the fingers. The author, being a convinced practitioner, took the liberty and did not delve into the endless and unconvincing theoretical discussions in order to realize the usual calculations (in the sense that all known and generally accepted formulas are used) to quantify the amount of information for several examples.

What do the measurements of the fundamental constants and the Maxwell's demon have in common? In fact, a little. Adapting the $\mu_{\mathrm{SI}}$-hypothesis, which was used in recent years to test the achievable relative uncertainty in measuring fundamental constants, we are developing a way to better understand specific problems that are closed to the Maxwell problem.

In one of his versions, the standard Maxwell's demon is a very small intellectual being endowed with free will, and a fairly subtle tactile and perceptive organization to enable him to observe and influence individual molecules of matter. In Maxwell's thought experiment, two gas chambers, maintained at equal temperatures, are separated by an adiabatic wall with a small hole and a gate that the demon opens and closes. Observing the speed of individual molecules, the demon selectively opens and closes the gate to quickly detach from slow molecules, creating a clean temperature difference between the two chambers. Thus, as the collisions with the shutter are elastic, and moving the shutter is frictionless, no work is performed by the demon. The temperature difference that develops could be exploited by a conventional heat engine to extract work, in violation of second law of thermodynamics.

Various researchers suggested different ways by which a demon could select particles in a reversible manner. Leó Szilárd in 1929 [34] argued that the demon must consume energy in the act of measuring the particle speeds and that this consumption will lead to a net increase in the system's entropy. In fact, Szilárd formulated an equivalence between energy and information, and calculated that $k_{\mathrm{b}} \cdot \theta \cdot \ln 2$ is both the minimum amount of work needed to store one bit of binary information and the maximum that is liberated when this bit is erased, where $\theta$ is the temperature of the storage medium. Through latest publications $[35,36,37,38]$, one must remember [39], in which there was finally clarified that the demon's role does not contradict the second law of thermodynamics, implying that we can, in principle, convert information to free energy. Toyabe et al in [40] showed that since the energy transformed from the information is compensated by the cost of the demon's energy for manipulating information, the second law of thermodynamics is not violated when a general system involving both a particle and a demon is considered. In the proposed research system, the demon consists of macroscopic devices, such as computers. The microscopic device receives energy due to the energy consumption of the macroscopic device. In other words, using information as an energy transfer medium, this transformation of information into energy can be used to transfer energy to nano machines, even if they cannot be directly controlled. In [41] there was declared that the 
Maxwell's demon can be converted into free energy by one bit of information obtained by measurement. The authors implemented an electronic Maxwell's demon based on a one-electron unit operating as a Szilard engine, where $k_{\mathrm{b}} \cdot \theta \cdot \ln 2$ heat is extracted from the reservoir at a temperature $\theta$ by one bit of generated information. The information was encoded in the position of an additional electron in the box. The authors provided, to their knowledge, the first demonstration of extracting nearly $k_{b} \cdot \theta \cdot \ln 2$ of work for one bit of information.

After 150 years a satisfactory additional solution of this paradox can be given [7]. In order to prevent the violation of the second law, one must assume that the demon is a conscious observer with knowledge, experience and intuition. Then, before performing any actions, in order to know the velocity of every molecule in the box, he must compose a mental model of the experiment, with no disturbances being brought into the box. In turn, the demon for the development of the model will take advantage of the already well-known International system of units (SI). When modeling a particle movement, the demon may choose quantities, for example, velocity, mass, angle of motion of the particle with respect to the shutter, temperature that may substantially differ from those chosen by another demon, as happened, for example, during the study of electrons that behave like particles or waves. That is why SI can be characterized by equally probable accounting of any quantity chosen by the demon. In this case, the total number of possible dimensionless criteria $\mu_{\mathrm{SI}}$ of $\mathrm{SI}$ with the seven base quantities $L, M, T, I, \Theta, J$ and $F$ could be calculated (7)

$$
\begin{aligned}
& \boldsymbol{\mu}_{\mathrm{SI}}=38,265, \\
& \boldsymbol{\Delta} \mathbf{A}^{\prime}=\mathbf{Q} \cdot\left(\mathbf{H}_{p r}^{\prime}-\mathbf{H}_{p S}^{\prime}\right)=1 \cdot\left[\boldsymbol{k}_{b} \cdot \ln \boldsymbol{\mu}_{S I}-\boldsymbol{k}_{b} \cdot \ln \left(\boldsymbol{z}^{\prime}-\boldsymbol{\beta}^{\prime}\right)\right]==\boldsymbol{k}_{b} \cdot \ln \left[\boldsymbol{\mu}_{S I} /\left(\mathbf{z}^{\prime}-\boldsymbol{\beta}^{\prime}\right)\right]
\end{aligned}
$$

where $\Delta \mathrm{A}^{\prime}$ is the a priori amount of information pertaining to the observed object due to the choice of the COP.

Following the same reasoning, one can calculate the a priori amount of information $\Delta \mathrm{A}^{\prime \prime}$, caused

$$
\boldsymbol{\Delta A} "=\boldsymbol{k}_{b} \cdot \ln \left[\left(\boldsymbol{z}^{\prime}-\boldsymbol{\beta}^{\prime}\right) /\left(\mathbf{z}^{\prime \prime}-\boldsymbol{\beta} "\right)\right]
$$

where $\Delta \mathrm{A}^{\prime \prime}$ cannot be defined without declaring the chosen $\operatorname{COP}\left(\Delta \mathrm{A}^{\prime}\right) ; z^{\prime \prime}$ is the number of physical dimensional quantities recorded in a mathematical model and $\beta^{\prime \prime}$ is the number of the base quantities recorded in a model of box.

$$
\Delta \mathrm{A}_{\mathrm{E}}=\Delta \mathrm{A}^{\prime}+\Delta \mathrm{A}^{\prime \prime}=\boldsymbol{\kappa}_{b} \cdot \ln \left[\boldsymbol{\mu}_{\mathrm{SI}} /\left(\boldsymbol{z}^{\prime \prime}-\boldsymbol{\beta}^{\prime \prime}\right)\right]
$$

where $\Delta A_{E}$ is measured in units of entropy [42], $z^{\prime \prime}$ is the number of physical dimensional quantities recorded in the mathematical model, $\beta^{\prime \prime}$ is the number of the base dimensional quantities recorded in a model.
A minimal amount of information $\triangle \mathrm{A}_{E}$ about the observed modeled box is calculated according to the following:

$$
\Delta \mathbf{A}_{\mathrm{b}}=\ln \left[\boldsymbol{\mu}_{\mathrm{SI}} /\left(\boldsymbol{z}^{\prime \prime}-\boldsymbol{\beta} "\right)\right] / \ln 2 \text { (bits). }
$$


Taking into account that $\mu_{\mathrm{SI}}=38,265$ and suppose $z^{\prime \prime}-\beta^{\prime \prime}=1$ (one can choose a larger number of dimensionless criteria, but this does not affect the course of further reasoning and conclusions, see Table 1), one can calculate the minimum boundary of the motion blur of a particle in the eyes of a demon

$$
\Delta \mathbf{A}_{\mathrm{b}}=\ln [38,265 / 1] / 0.6931472 \approx 11 \text { (bits). }
$$

Thus, equation (24) contains a very strong hint that the demon is not able to clearly distinguish the exact state of a large number of particles. There are no glasses that could correct the sight of the demon. This closes the possibility of developing a device that could distinguish between fluctuations in individual particle velocities. Hence it is clear that any material physical device, in comparison with a mental thought experiment (conscious, without a material shell by a demon), will require much more information and energy for the release of any gate movement.

$$
\Upsilon_{\text {bp }} \leq\left(2 \cdot \pi \cdot r_{p} \cdot E_{p}\right) /(\hbar \cdot c \cdot \ln 2)=270(\text { bit }) .
$$

where $r_{b p}$ is the information amount expressed in bits and corresponding to the photon's sphere; $r_{p}$ is the radius of photon expressed in meters, $2.8 \cdot 10^{-15} \mathrm{~m}$ [44]; $\mathrm{C}$ is the light speed, $\mathrm{C}=299,792,458 \mathrm{~m} / \mathrm{s}, \mathrm{\hbar}$ is the reduced Plank constant, $\hbar=1.054572 \cdot 10^{-34} \mathrm{~m}^{2} \cdot \mathrm{kg} \cdot \mathrm{s}^{-1}$, $\ln 2=0.693147, \pi=3.141593$.

Thus, the minimum boundary of the motion blur of the particle in the eyes of the demon (in bits) is much less than the information contained in the photon (270 bit » 11 bit). However, this fact does not in any way allow us to state that the demon, after preliminary modeling, will be able to carry in one direction particles moving at high speed, and in the other way - particles having a low speed, thereby violating the second law of thermodynamics. On the contrary, the demon will need information through a measuring device that is comparable in magnitude to the information inherent in the particle. This, in turn, will require the performance of work, which will lead to an increase in entropy in the total volume of the casket.

The proposed approach provides only a hint of how much information a demon and the observed particle have before starting any action with a system box-demon or about "uncertainty" in the mind of someone about to receive a message [46].

\section{d) Universe information associated with energy}

In connection with the foregoing, there is an amazing possibility (and for the readers maybe a very controversial one) of applying the results obtained in analyzing the status of the miniature demon Maxwell to the problems associated with clarifying the energy of the observed universe.

Experiments and theories developed in theoretical physics over the past decades have demonstrated the significant role of information, the amount of which physicists usually identify with entropy, but which can be more general when used to explain the emerging complexity of the universe. One of the most attractive features of the Bekenstein formula [47] is that it allows us to compose an idea of the possible connection between energy and information contained in the universe.

For this purpose, let us recall [47], in which it was proved that the amount of information of any physical system must be finite if the space of object and its energy are finite. In informational terms, this boundis given by

$$
r_{\mathrm{b}} \leq(2 \cdot \pi \cdot R \cdot E) /(\hbar \cdot c \cdot \ln 2),
$$

where $r_{b}$ is the information expressed in the number of bits contained in the quantum states of the chosen object sphere. The In2 factor (approximately 0.693149) comes from defining the information as the natural logarithm of the number of quantum states, $R$ is the radius of an object sphere that can enclose the given system, $E$ is the total mass-energy, including rest masses, $\hbar$ is the reduced Planck constant, and $c$ is the speed of light.

Further, the Landauer principle [39], which is applicable to all systems in nature, asserts that the minimum amount of energy required to destroy one bit of information is:

$$
k_{\mathrm{b}} \cdot \theta \cdot \ln 2 \text {, }
$$

where $\theta$ is the temperature in kelvins of environment.

It is important to note that the equivalent bit energy depends on the temperature of the described system. The average temperature of the universe today is approximately $\theta=2.73 \mathrm{~K}$ [48], based on measurements of cosmic microwave background radiation. Therefore, with a bit of imagination and an essential assumption, in order to transform $\gamma_{b}$ to termsof the ordinary energy $\Upsilon_{\mathrm{E}}$, one should multiple it by $k_{\mathrm{b}} \cdot \theta \cdot \ln 2$. 


$$
\Upsilon_{E}=\Upsilon_{\mathrm{b}} \cdot k_{\mathrm{b}} \cdot \theta \cdot \ln 2 \leq((2 \cdot \pi \cdot R \cdot E) /(\hbar \cdot c \cdot \ln 2)) \cdot k_{\mathrm{b}} \cdot \theta \cdot \ln 2,
$$

or

$$
\Upsilon_{E} / E \leq\left(2 \cdot \pi \cdot R \cdot \kappa_{b} \cdot \theta\right) /(\hbar \cdot C)
$$

Using the dimensional analysis, we verify the achieved dimension of equation (29).

$\operatorname{dim} \mathrm{R} \ni \mathrm{m} ; \operatorname{dim} \mathrm{k}_{\mathrm{b}} \ni \mathrm{kg} \cdot \mathrm{m}^{2} \cdot \mathrm{s}^{-2} \cdot \mathrm{K}^{-1} ; \operatorname{dim} \theta \ni \mathrm{K} ; \operatorname{dim} \hbar \ni \mathrm{m}^{2} \cdot \mathrm{kg} \cdot \mathrm{s}^{-1} ; \operatorname{dim} \mathrm{C} \ni \mathrm{m} \cdot \mathrm{s}^{-1} ;$

$$
\operatorname{dim}\left(\Upsilon_{E} / E\right) \ni \mathrm{m} \cdot \mathrm{kg} \cdot \mathrm{m}^{2} \cdot \mathrm{s}^{-2} \cdot \mathrm{K}^{-1} \cdot \mathrm{K} /\left(\mathrm{m}^{2} \cdot \mathrm{kg} \cdot \mathrm{s}^{-1} \cdot \mathrm{m} \cdot \mathrm{s}^{-1}\right)=1
$$

So, at least, from the point of view of the dimensional analysis there is not a fatal mistake.

Further, the age of universe $T_{\text {univ }}$ is about $13.7 \pm 0.13$ billion years or $4.308595 \times 10^{17} \mathrm{~s}[49]$.

$$
\mathrm{R}_{\text {univ }}=\mathrm{T}_{\text {univ }} \cdot \mathrm{C}=1.291684 \cdot 10^{26}(\mathrm{~m}) \text {. }
$$

It should be noted that there is no known boundary, that is, $R_{\text {univ }}$ is an approximate value. When people talk about the size of observable universe, this means the estimated distance to the most distant objects that we can see here. This does not mean that there is nothing further; it simply means that we do not see it.

$$
\begin{aligned}
& \Upsilon_{E} / E \leq\left(2 \cdot \pi \cdot R_{\text {univ }} \cdot K_{b} \cdot \theta\right) /(\hbar \cdot C)= \\
& =\left(2 \cdot 3.141593 \cdot 1.291684 \cdot 10^{26} \cdot 1.38064852 \cdot 10^{-23} \cdot 2.73\right) /\left(1.054572 \cdot 10^{-34} \cdot 299,792,458\right)= \\
& =30.590185 \cdot 10^{3} / 3.161527 \cdot 10^{-26}=9.6 \cdot 10^{29} \approx 10^{30}
\end{aligned}
$$

Thus, we have shown that the energy associated with information makes a significant contribution to the total energy of the universe. Of course, this (32) is a rough estimate. It is interesting to note that $10^{30}$ is much less than $10^{122}$. According to the holographic principle, the last huge number represents an upper bound on the information content of the universe[50]. Since information energy can make a significant contribution to the dark energy and dark matter of the universe, scientists need to study it more closely. Maybe this value $\left(10^{30}\right)$ can also be a signal of some kind of new interaction of matter and information.

Therefore, more is unknown than known. Besides this, it is a complete secret. But this is an important secret. The rest is everything on the Earth, everything that has ever been observed with all our instruments, all normal energy, is a meager part of the universe. Think about it, perhaps it is not a "normal" at all, since it is such a small part of the universe. But what kind of information is this? Perhaps information itself is a fundamental entity of the physical universe. Is it "ontological" - the real substance from which space, time and matter emerge? Or is it "epistemic" - something that only represents our state of knowledge about reality? Ultimately, information can be a key element in the constitution of physical reality and it is a decisive content in physical systems and technological processes. The explicit relationship between entropy and information, using the concept of objective quantitative information of Shannon, was formalized in [51], and this can be regarded as irrefutable confirmation of information as a physical entity.

Such a dramatic gap of $10^{30}$ between the amounts of energy associated with the ordinary matter and the energy due to information can be conditioned with the assumptions originally assumed: the universe is not a sphere; the average temperature of the universe can be much lower than the observed temperature; for the giant distance scale, the Landauer's limit is not satisfied.

The presented results (24), (25), (32) are simply a routine calculation by formulas known in the scientific literature. At the same time, only experts of quantum electrodynamics or the theory of gravity can "separate wheat from chaff". However, if the Bekenstein formula and the Landauer's limit have a physical explanation, perhaps the result (32) can be used to study the universe.

Additional explanation of how information acquires energy comes from the quantum theory of matter. In this theory, "empty space" is actually full of temporal ("virtual") particles that are constantly being formed, possessing certain information, and then disappear. But when we tried to calculate how much energy this information gives to the empty space, the answer turned out to be erroneous - wrong by a lot. The 
number of $10^{30}$ is too large. It is difficult to get such an answer. So, the mystery exists.

Another explanation of the significant magnitude of the energy corresponding to the information contained in the universe is that it is a new kind of field energy that fills the whole space. But if the information itself is the answer, we still do not know what it is, what it interacts with or how it exists in the universe. Thus, the mystery continues.

More speculatively, a last possibility is that Einstein's theory of gravity is not correct. That would affect the way that normal matter in galaxies behaved. This fact would provide a way to decide if the solution of the amount of information is a possible and inadmissible part of the new gravity theory or not. Thus, there are many questions, no answers. That is why; things are still not so bad as to expect improvement.

As an alternative to dark energy and dark matter, the energy due to information contained in the universe can serve as a fundamental component [52-55]. The giant difference between the two types of energy (32) makes it possible to assert that the universe is isotropic-the same in all directions-and homogeneous, without the regions of the cosmos, which have special, peculiar characteristics. Equation (32) cannot be an illusion caused by mathematics. It does not just come out by accident. Does this mean that our universe consists of information, and the associated energy is responsible for the inhibition of space and time and the accelerating expansion that we observe? It is difficult for the matter-of-fact physicist to agree with this point of view. Maybe there are better ideas? It is tempting to look for links and analogies, even if they are at first considered bad for discussion. Perhaps in the future these two problems are not as fragmented as they might seem. Formulating a problem that at first glance seems completely extravagant can sometimes, with further reflection, acquire real meaning and become very meaningful for the further development of science.

\section{Discussion}

Apparently, the application of information theory to calculate measurement uncertainty will be unnatural for some readers. If you ask why such cases are generally considered, then instead of the answer it is useful to recall the English anecdote about the doctorpathologist. One of his students said that he does not see the benefits of pathological physiology, because it is so unnatural. The doctor called him a fool and added: Only by studying pathology, it is possible to establish true health conditions.

At present, the term "comparative uncertainty", as well as "information contained in the model", "change in entropy in a mental experiment," obtained the rights of citizenship. Physical systems, which include such elements, are systems used both in experimental physics and in any technological processes. In this paper, various applications of the information-oriented method are presented. The $\mu_{\mathrm{SI}}$ hypothesis made it possible to establish the fact that scientists may approach, but never reach, the comparative uncertainty corresponding to the chosen COP. Regardless of the implementation of super power computers, brilliant modern data processing methods and unique test benches, comparative uncertainty, even with the required number of dimensionless criteria, will be unattainable. In addition, the $\mu_{\mathrm{SI}}$ hypothesis made it possible to judge the appropriate limit of the accuracy of measurements in each individual case.

Under the proposed approach, for each mathematical model of physical law there is an uncertainty, which initially, before the full-scale experimental studies, or computer simulations, describes its proximity to the examined physical phenomenon or process. This value is called the comparative uncertainty. It depends only on the number of selected quantities and the observation interval of the selected primary quantity. One of the interesting features of the proposed hypothesis is that the minimum achievable comparative uncertainty is not constant and varies depending on the class of phenomena choice. Moreover, theory can predict its value. In particular, this means that when switching from a mechanistic model $(L M T)$ to COP with a larger number of the base quantities, this uncertainty grows. This change is due to the potential effects of the interaction between the increased number of quantities that can be taken into account or not taken into account by the researcher.

On the one hand, well-known physical laws are valid in a certain area and served as a reliable tool in everyday life. At the same time, taking into account the experience of the creation of special relativity theory, we know that the achieved accuracy of the description of the world is not satisfactory. On the other hand, fundamental physical constants currently measured with high accuracy. However, it is not sufficient to be able to modify the International system of units (SI). The proposed approach allows us to estimate the limits of our knowledge and to reveal an insurmountable barrier for identifying compliance of model and the object studied. A clear evidence of this is a possibility to estimate the minimum attainable value of the relative uncertainty for the gravitational constant, Planck's constant, the fine structure constant, Boltzmann's constant, Avogadro's constant, especially considering that the predictions do not contain quantities that can be chosen intuitively or based on statistical methods.

\section{Conclusions}

In addition to the relative uncertainty analysis, the introduced approach could enable new methodology that will help the additional monitoring the measurement accuracy of fundamental physical 
constants. The use of the $\mu$-hypothesis only limits the domain of applicability of measurement theory for uncertainties that are much larger than the uncertainty of the physical-mathematical model due to its finiteness.

By introducing the comparative uncertainty concept along with known physical laws, we can verify required relative uncertainties values of fundamental physical constants that must be recommended for identifying concrete ways in perfecting SI. The suggested approach is a mathematical tool that allows describing a physical system with the lowest uncertainty, which is a surprisingly simple relation.

If the measure of the beauty of the theory is the ratio of the number of things that it explains, how many assumptions it makes for their explanation, then the information-oriented approach seems very promising. $\mu_{\mathrm{SI}}$ hypothesis does refer to a real place of the surrounding world. It might be applicable to experimental verification. In general, it is available when the researcher has all the information about the uncertainty interval of the main quantity. Moreover, $\mu_{\mathrm{SI}}$ hypothesis provides new functionalities useful for micro- and macro-physics including engineering, astronomy, and quantum electrodynamics. The comparative uncertainty can be a peculiar metric for the assessing the measurement accuracy of physical laws and fundamental physical constants.

The information-oriented approach, in particular, IARU, makes it possible to calculate with high accuracy the relative uncertainty, which is in a good agreement with the recommendations of CODATA. The principal difference of this method, in comparison with the existing statistical and expert methodology of CODATA (actually all statistical methods are unreliable some more and some less [56]), is the fact that the information method is theoretically justified.

There is a weak tension between some (but not all) astrophysical measurements and cosmological conclusions. There are several ways to look at it. First, one or more methods are now limited to systematic; in other words, the subject is limited to accuracy, not precision, and that close attention to the underestimated systematic will lead to a convergence of values in the next few years. Secondly, it is possible that the new physics is involved outside the change in the index of dark energy. New physical experiments will require a relative uncertainty of $1 \%$ or less of the definitions of $\mathrm{H}_{0}$, given the current state of play in cosmology.

Significant differences in the values of the comparative uncertainties achieved in the experiments of measuring $\mathrm{H}_{0}$ and calculated in accordance with the IACU can be explained as follows. The very concept of comparative uncertainty, within the framework of the information approach, assumes an equally probable account of various quantities, regardless of their specific choice by scientists when formulating a model for measuring a particular fundamental constant. Based on their experience, intuition and knowledge, the researchers build a model containing a small number of quantities, and which, in their opinion, reflects the fundamental essence of the process under investigation. In this case, many phenomena, perhaps not significant, secondary, which characterized by specific quantities, are not taken into account.

For example, when measuring the value of the Hubble constant by $\mathrm{CMB}\left(\mathrm{CoP}_{\mathrm{SI}} \equiv L M T\right)$, some assumptions are advanced: dynamic dark energy is modeled as an ideal fluid; flat universe; a fixed cosmological model. Thus, the developers do not take into account that: the recognition of dark energy in the form of an ideal fluid is physically inconsistent and does not adequately approach the evolution of dark energy; in the real world the universe is not flat; the expected model may differ slightly from the models taken for analysis, etc. In this case, we get a paradoxical situation. On one side, different groups of scientists dealing with the problem of measuring a certain fundamental constant and using the same method of measurement "learn" from each other and improve the test stand to reduce uncertainties known to them. This is clearly seen using the IARU method: when measuring, for example, $\mathrm{h}, k_{\mathrm{b}}, \mathrm{N}_{\mathrm{A}}, \alpha, \mathrm{G}$, all the relative uncertainties are very consistent, especially for measurements made in recent years. However, ignoring a large number of secondary factors, which are neglected by experimenters, leads to a significant variance in the comparative uncertainties calculated by the IACU method.

Obviously, the coordination of a probabilistic subatomic world with a macroscopic everyday world is one of the greatest unsolved problems in physics. The use of the $\mu_{\mathrm{SI}}$ hypothesis opens the possibility of combining these two worlds: from Maxwell's demon to cosmology and astrophysics.

$\mu_{\mathrm{SI}}$ hypothesis allows to obtain the entropy cost associated with the acquisition of the demon information. Any demon, no matter how smart it is, must perform measurements. Certainly, when creating a model for the separation of particles, it is necessary to consider in detail the constitution of a rational being. The possession of information can indeed be regarded as a decrease in entropy. However, in the case of mental modeling, obtaining information does not require the dissipation of heat, and there is no threat to the generalized form of the second law.

Mental modeling requires us to say something about the demon itself as a physical being. A demon can perform a modelling without energy dissipation. This fully corresponds to the position of the Brillouin. He characterized the information as "connected" if it was embodied in states of the physical device, but he bluntly 
stated that information contained only in the mind is "free", and not "connected".

Now the connection between entropy and information becomes more understandable. When the demon leaves the system, he can be viewed as an agent that has information about the system. Uncertainty in the description of the system can be considered as a lack of knowledge of the demon about the exact state of the system. If the demon has more information, the system's entropy is smaller. However, once the demon can obtain information without dissipation, the system's entropy decreases, and the only compensation appears to be an increase in the uncertainty of the state of the demon itself.

\section{References Références Referencias}

1. Henrion, M. Fischhoff, B. (1986). Assessing uncertainty in physical constants, Am. J. Phys. 54(9), 791-798. goo.gl/nfMcNW.

2. Kušnerová, M. (2013). A Proposal for simplifying the method of evaluation of uncertainties in measureement results, Measurement science review, 1, 1-6. https://content.sciendo.com/view/journals/msr/13/1/ article-p1.xml.

3. Finkelstein, L. (1975). Representation by symbol systems as an extension of the concept of measurement, Kybernetes, 4(4), 215-223.http://scihub.tw/10.1108/eb005397.

4. Hartley, R. V. (1928). Transmission of information, Bell System technical journal, 7(3), 535-563.http:// keszei.chem.elte.hu/entropia/Hartley1928text.pdf.

5. Van Fraassen, B. C. (2008). Scientific Representation: Paradoxes of Perspective, Oxford: Oxford University Press. 141-185. http://strange beautiful.com/other-texts/fraassen-sci-rep.pdf.

6. Mari, L. (1999). Notes towards a qualitative analysis of information in measurement results, Measurement, 25(3), 183-192. http://sci-hub.tw/10. 1016/S0263-2241(99)00002-0.

7. Menin, B. (2017).Information Measure Approach for Calculating Model Uncertainty of Physical Phenomena, American Journal of Computational and Applied Mathematics, 7(1), 11-24.https://goo. $\mathrm{gl} / \mathrm{m} 3 \mathrm{ukQi}$.

8. Menin, B. (2017). Preferred physical-mathematical model of the cold energy storage system. Applied Thermal Engineering, 112, 1020-1026. http://scihub.tw/10.1016/j.applthermaleng.2016.10.128.

9. Menin, B. (2016). Accuracy of Predictions of Ice Slurry Properties and Technical Characteristics of Machines Producing It, American Journal of Computational and Applied Mathematics, 6(2), 7481.http://goo.gl/QkLN12.

10. Menin B. (2015). Accuracy limits of modelling predictions of multiquantity heat and mass transfer
Processes, Int. J. Mathematical Modelling and Numerical Optimization, 6(4), 321-336.

11. Sonin, A. A. (2001). The physical basis of dimensional analysis, 2nd ed. Department of Mechanical Engineering, MIT, Cambridge. goo.gl/ 2BaQM6.

12. NIST Special Publication 330 (SP330) (2008). The International System of Units (SI), 2008.http://goo. $\mathrm{gl} / 4 \mathrm{mcVwX}$.

13. Jakulin, A., Symmetry and information theory, 1-20, 2004. goo.gl/QGBVoU.

14. Sedov, L. I. (1993). Similarity and Dimensional Methods in Mechanic, CRC Press, Florida.

15. Brillouin, L. (1964). Scientific uncertainty and information, Academic Press, New York.

16. Balalaev, V. A., Slayev, V. A., Sinyakov, A. I. (2005). Potential accuracy of measurements: Scientific publication -Textbook / Edited by Slayev, V. A., ANO NPO Professional, St. Petersburg, In Russian. http:// www.vniim.ru/files/PotTochlzm.pdf.

17. Hinshaw, G. et al.(2009). Five-Year Wilkinson Microwave Anisotropy Probe (WMAP) Observations: Data Processing, Sky Maps, and Basic Results, the Astrophysical Journal Supplement Series, 180, 225-245. https://goo.gl/VBp3er.

18. Riess, A. G. et al. (2011). A 3\% Solution: Determination of the Hubble Constant with the Hubble Space Telescope and Wide Field Camera 3, Astrophys. J., 730,119. https://arxiv.org/pdf/1103. 2976.pdf.

19. Jarosik, N. et al. (2011). Seven-year Wilkinson microwave anisotropy probe (WMAP) observations: sky maps, systematic errors, and basic results, The Astrophysical Journal Supplement Series, 192(14), 1-15. https://goo.gl/ScM4uV.

20. Bennett, C. L. et al. (2012). Nine-Year Wilkinson Microwave Anisotropy Probe (WMAP) Observations: Final Maps and Results. https://arxiv.org/abs/12 12.5225.https://goo.gl/SBr4Hc.http://sci-hub.tw/10. 1088/0067-0049/208/2/20.

21. Efstathiou, G. (2014). $H_{0}$ revisited, Monthly Notices of the Royal Astronomical Society, 440(2), 1138-1152. http://sci-hub.tw/10.1093/mnras/stu278.

22. Bennett, C. L., Larson, D., Weiland, J. L., Hinshaw, G. (2014). The 1\% concordance Hubble constant, The Astrophysical Journal, 794(135), 1-8.http://iop science.iop.org/article/10.1088/000437X/794/2/135/ pdf.

23. Cheng, C., QingGuo, H. (2015). An accurate determination of the Hubble constant from baryon acoustic oscillation datasets, Science China: Physics, Mechanics \& Astronomy, 58(9), 1-6.http:// sci-hub.tw/10.1007/s11433-015-5684-5.

24. Planck collaboration, Planck 2015 results. XIII, Cosmological parameters Astronomy \& Astro- 
physics, 594,id.A13,1-63.http://sci-hub.tw/10.1051/ 0004-6361/201525830.

25. Yu, H., Ratra, B., Wang, F.-Y. (2018). Hubble Parameter and Baryon Acoustic Oscillation Measurement Constraints on the Hubble Constant, the Deviation from the Spatially Flat $\Lambda$ CDM Model, the Deceleration-Acceleration Transition Redshift, and Spatial Curvature, The Astrophysical Journal, 856(1),3,1-11. http://sci-hub.tw/10.3847/1538-4357/ aab0a2.

26. Benson, B. (2018). The Hubble Constant from the Cosmic Microwave Background, American Physical Society Meeting. Presentation. https:// ab sups loa ds.aps.org/presentation. cfm? pid $=14044$.

27. Menin, B. (2018). Novel Method for Calculating the Measurement Relative Uncertainty of the Fundamental Constants, American Journal of Computational and Applied Mathematics, 8(4), 7079. http://article.sapub.org/10.5923.j.ajcam.201808 04.02.html.

28. Newell, D. B. et al. (2017). The CODATA 2017 Values of $h, e, k$, and NA, Metrologia, 54, 1-6. http://sci-hub.tw/10.1088/1681-7575/aa950a\# .

29. Wood, B. M., Sanchez, C. A., Green, R. G., Liard, J.O. (2017). A summary of the Plank constant determinations using the NRC Kibble balance, Metrologia, 54, 399-409.http://sci-hub.tw/10.1088/ 1681-7575/aa70bf.

30. Azuma, Y. et al. (2015). Improved measurement results for the Avogadro constant using a $28 \mathrm{Si}$ enriched crystal, Metrologia, 52(2), 360-375. https://goo.gl/PURKaG.

31. Kirakosyan, G. S. (2010). The correlation of the fine structure constant with the redistribution of intensities in interference of the circularly polarized Compton's wave, Gen. Phys., 1-7. http://nt.ru/tp/ng/fs1.pdf.

32. Mohr, P. J. et al. (2016). CODATA Recommended Values of the Fundamental Physical Constants: 2014. Reviews of Modern Physics, 88. https://goo. gl/O0x4cv.

33. Oliver, T. A., Terejanu, G., Simmons, C. S., Moser, R. D. (2015).Validating predictions of unobserved quantities, Computer Methods in Applied Mechanics and Engineering, 283, 1310-1335.http:// sci-hub.tw/10.1016/j.cma.2014.08.023.

34. Szilárd, L. (1929). On the decrease of entropy in a thermodynamic system by the intervention of intelligent beings. Z. Phys. 53, 840-856. http:// www.sns.ias.edu/ tlusty/courses/InfolnBio/Papers/ Szilard1929.pdf.

35. Bennett, C. H. (1982). The thermodynamics of computation, Int. J. Theor. Phys. 21, 905-940.

36. Sagawa, T., Ueda, M. (2009). Minimal energy cost for thermodynamic information processing: Measurement and information erasure, Phys. Rev.
Lett. 102, 250602. http://sci-hub.tw/10.1103/Phys RevLett.102.250602

37. Leff, H. S., Rex, A. F. (2003). Maxwell's Demon 2: Entropy, Classical and Quantum Information, Computing (Institute of Physics Publishing, 2003).

38. Maruyama, K., Nori, F., Vedral, V. (2009). The physics of Maxwell's demon and information, Rev. Mod. Phys. 81, 1-23.

39. Landauer, R. (1961). Irreversibility and heat generation in the computing process, IBM J. Res. Dev. 5, 183-191. https://goo.gl/LYQEU8.

40. Toyabe, S., Sagawa, T., Ueda, M., Muneyuki, E., Sano, M. (2010). Experimental demonstration of information-to-energy conversion and validation of the generalized Jarzynski equality, Nature Physics, Letters, 1-5. http://cat.phys.s.u-tokyo. ac.jp/ ueda/ ToyobeNPHYS.pdf.

41. Koskia, J. V., Maisia, V. F., Pekolaa, J. P., Averind, D.V. (2014). Experimental realization of a Szilard engine with a single electron, PNAS, 111(38), 13786-13789. http://www.pnas.org/content/111/38/ 13786.

42. Menin, B. M. (2015). Possible limits of accuracy in measurement of fundamental physical constants. International Referred Journal of Engineering and Science, 4(8), 8-14. http://goo.gl/HjYBOs.

43. Volkenstein, M. V. (2009). Entropy and Information. Birkhauser Verlag AG, Basel-Boston-Berlin. https:// goo.gl/eHhRvd.

44. Liu, S.-L. (2017). Electromagnetic fields, size, and copy of a single photon, 1-4.https://arxiv.org/ftp/ arxiv/papers/1604/1604.03869.pdf.

45. Menin, B. (2018). Bekenstein-Bound and Information-Based Approach, Journal of Applied Mathematics and Physics, 6(8), 1675-1685. doi: 10.4236/jamp.2018.68143.

46. Schement, J. R., Ruben, B. D. (1993). Information Theory and Communication, Transaction Publishers, 4, 43-53.

47. Bekenstein, J. D. (1981). A Universal Upper Bound on the Entropy to Energy Ratio for Bounded System. Physical Review D, 23, 287-298. http://scihub.tw/10.1103/PhysRevD.23.287.

48. Pettini, M. (2015). Introduction to Cosmology Lecture 7, 1-11. https://www.ast.cam.ac.uk/ pettini/ Intro\%20Cosmology/Lecture07.pdf.

49. WMAP Science Team Cosmology: The Study of the Universe: NASA's Wilkinson Microwave Anisotropy Probe. (2011). https://map.gsfc.nasa.gov/ universe/ WMAP_Universe.pdf.

50. Davies, P.C.W. (2007). The implications of a holographic universe for quantum information science and the nature of physical law, 1-15. http://power.itp.ac.cn/ mli/pdavies.pdf.

51. Zurek, W. H. (1989). Algorithmic randomness and physical entropy. Physical Review A, 40(8), 4731-4751. 
52. Lloyd, S. (2002). Computational Capacity of the Universe, Physical Review Letters, 88(23), 1-4. http://fab.cba.mit.edu/classes/862.16/notes/comput ation/Lloyd-2002.pdf.

53. Mukhopadhyay, A. K. (2008). A Radical view of Information on its nature and science, Frontier Perspectives, 16(2), pp. 19-29. https://akmu khopa dhyayconsciousness.com/pdf/LINK6.pdf.

54. Haranas, I., Gkigkitzis, I. (2014). Bekenstein Bound of Information Number $\mathrm{N}$ and its Relation to Cosmological Parameters in a Universe with and without Cosmological Constant, 1-8.https://scihub.se/10.1142/S0217732313500776.

55. Padmanabhan, T., Padmanabhan, H. (2017). Cosmic information, the cosmological constant and the amplitude of primordial perturbations, Physics Letters B, 773, 81-85.https://sci-hub.se/10.10 16/j.physletb.2017.07.066.

56. Burgin, M. (2003). Information Theory: a Multifaceted Model of Information, Entropy, 5, 146-160. http://www.mdpi.org/entropy/papers/e50 20146.pdf. 